\title{
Effects of Directional Antennas on Outband D2D mmWave Communications in Heterogeneous Networks
}

\author{
Romain Chevillon, Guillaume Andrieux, Romain Négrier and Jean-François \\ Diouris \\ Université Bretagne Loire, Université de Nantes, IUT de La Roche-sur-Yon, \\ UMR6164: Institute of Electronics and Telecommunications of Rennes (IETR), \\ 18, boulevard Gaston-Defferre, 85035 La Roche-sur-Yon cedex, France
}

\begin{abstract}
Device-to-Device (D2D) communications are considered as a keystone of the fifth generation wireless technology $(5 \mathrm{G})$. This new approach is very promising in terms of energy and spectrum efficiency. However, the integration of such communications in a typical cellular network increases inevitably the amount of interference. Several researches propose to lower the interference thanks to either sharing the cellular spectrum intelligently, or using non-cellular bands for D2D links. In this paper, we focus on the latter opportunity, and consider that the D2D communications are used with millimeter waves (mmWaves).

For what comes to modeling a D2D-enabled (D2D-e) network, many works propose to use stochastic geometry so as to evaluate the impact of interference and noise on the various links. In this work, we aim to analyze the SINR and the average data rate of Outband D2D links for user equipments (UEs) with conventional omnidirectional antennas and with various directional mmWave antennas: patch antennas, horn antennas and uniform linear array antennas. Analytical and empirical evaluations of the Signal-to-Interference-plus Noise Ratio (SINR) are made with stochastic geometry. We propose to discuss the advantages and drawbacks of directional mmWave antennas in Outband D2D for various antenna designs, and their interest in various environments.
\end{abstract}

Keywords: Device-to-device communication, heterogeneous networks, directional antennas, millimeter wave communication, stochastic geometry.

Preprint submitted to AEU - International Journal of Electronics and CommunicationsAugust 20, 2018 


\section{Introduction}

The increasing demand on proximity services and on faster data rates has motivated numerous researches on device-to-device (D2D) communications [1]. These new means of communications are considered as a very promising new

5 technology and a keystone of the intended fifth generation wireless technology (5G). Besides proximity services, D2D communications also allow to lower the battery usage for short distance transmissions, and can be used for information relaying from a device to another device, or from a device to a base station (BS), as well as for direct communications between devices 2. This type of communications requires a synchronization between devices, made either by the devices themselves (via tokens called "beacons"), or by the base stations [3, 4]. The use of D2D communications with mmWaves can improve considerably the performances of the network. For instance, in [5], the authors propose a joint transmission scheduling scheme for the radio access and backhaul of small cells

15 in the mmWave band that fully exploits the spatial reuse in a mmWave network. This protocol outperforms other proposed protocols in terms of data rate and delay. D2D communications in mmWave channels can also be used for multicast, as in 6. In this work, the authors exploit the physical proximity of users to improve multicast performance, and show high performance in terms of energy efficiency.

\subsection{Related Works}

In terms of spectrum sharing, D2D communications are mainly proposed to use the whole cellular spectrum (i.e. underlay Inband D2D) [7, 8. Nevertheless, in order to avoid the interference between typical and D2D communications, some works propose to dedicate a part of the cellular spectrum for only D2D communications (i.e. overlay Inband D2D) [9, 10]. Another approach based on unlicensed bands for D2D communications (i.e. Outband D2D) is also considered 11.

Although biological safety [12] and channel behavior [13, 14, of millimeter waves (mmWaves) are not totally defined at the moment [15], the mmWave 
spectrum is a very interesting option for the next generation of wireless communications. Indeed, mmWave spectrum can support hundreds of times more capacity than the current cellular spectrum [1, 16. In [17, the distinct propagation characteristics of mmWave bands and LTE bands are exploited to maximize the overall network data rate in an heterogeneous network configuration. The authors of [18] give measurement and radio wave propagation info for mmWave channels. In [19, the authors propose a system architecture based on mmWaves and LTE. Their method introduces an efficient resource sharing scheme that allows D2D links without interference. The authors of [11] propose to study the propagation of the mmWave spectrum (especially ISM bands in $24 \mathrm{GHz}$ and $61 \mathrm{GHz}$ ) using ray tracing models in urban environments. Their results prove that mmWaves for D2D communications are highly feasible, but only with the help of beam forming and beam switching. Indeed, these two methods permit to leverage the reflections and refractions due to urban structures. Besides, the authors of [1] and 20] reveal that the common buildings are very resistant to the penetration of mmWaves.

In [13, the authors present their results on channel measurement campaigns in mmWave bands, and develop advanced beamforming algorithms that demonstrate that mmWaves can be very promising for $5 \mathrm{G}$ cellular systems. The prototypes developed in their work include antenna arrays that are quite close to some of the antennas analyzed in our work. The authors of [21] integrate realistic antenna gain profiles for patch and horn antennas (these types of antennas are among the types introduced in our work) in a cellular network so as to enable wireless power transfers. The theoretical calculations are made with the help of stochastic geometry, and lead to encouraging results in terms of coverage and energy harvesting.

In terms of modeling, most works on D2D-enabled (D2D-e) networks use stochastic geometry to analyze power consumption, spectrum sharing and other characteristics [22, 23]. In particular, the use of Point Processes such as Poisson

${ }_{60}$ Point Processes (PPP) is significant in the works dealing with this topic. In [24], the authors introduce an empirical and analytical model of a D2D-e network, 
and demonstrate the signal-to-interference-plus-noise ratio (SINR) calculations related to their marked-PPP model. In [9, the authors adapt the results from 24] with the 3GPP propagation model. In 25], the authors use stochastic geometry to validate new spectrum access policies that may reduce interference. A D2D cluster model is introduced in [26, where the locations of the devices are modeled by a Poisson cluster process in which the parent point process is modeled by a PPP. The authors of [27] introduce a system model in which the locations of base stations and users are modeled by two PPP. In their work, they analyze the cellular network performance during massive infrastructure failure.

\subsection{Contributions and Organization}

In the previously cited works, mmWaves-based D2D communications are either modeled by considering only one specific macrocell, or studied in-situ without any deep theoretical approach. Moreover, the related works dealing with stochastic geometry for D2D links only focus on Inband D2D communications. In this paper, we propose to deeply analyze the advantages and the drawbacks of the use of mmWave directional antennas for Outband D2D communications. We focus on five types of antennas: omni-directional antennas (i.e. non-directional antennas), patch antennas, horn antennas, uniform linear array

80 (ULA) with two elementary antennas, and ULA with five elementary antennas. We propose to calculate theoretically and compare the SINR and the average data rates for each antenna type by modeling the $\mathrm{D} 2 \mathrm{D}$-e network, and validating this model. Then, the design of mmWave directional antennas is analyzed in a probabilistic view, so as to incorporate such antennas in our model.

The contributions of this paper are as follows:

- A D2D-e network model that considers channel inversion is introduced, which is more realistic than the use of a constant transmit power. Modern communications use power control techniques to mitigate energy consumption and interference. Channel inversion permits to adapt the transmission power relative to the link distance, the path-loss exponent and the signal-to-noise ratio (SNR). 
- Real radiation patterns (and not sectored patterns) are considered for the different types of directional antennas, which is more realistic. These real radiation patterns are incorporated probabilistically in the system model.

\section{System Model}

\subsection{Network Model}

We consider a hybrid system comprising both cellular and D2D communications. As explained in [28, 29] and shown in Figure 1, all the BSs are placed randomly according to a PPP $\Phi_{B}$, with a density $\lambda_{B}$. The set of BSs forms a Voronoi tessellation, where the Voronoi cells constructed from the PPP correspond to the coverage regions of the BSs. The user equipments (UEs) are distributed as pairs over the space with a dipole model as depicted in [29]. 
Then, the UEs are modeled by an independently marked PPP denoted as

$$
\tilde{\Phi}=\left\{\left(X_{i}, \delta_{i}, L_{i}, P_{i}, \theta_{i}\right)\right\},
$$

120

\subsection{Interferences and SINR Characterization}

We consider a $\mathrm{D} 2 \mathrm{D}$ pair $\mathrm{DP}_{i}$ comprising a transmitter $\mathrm{D}_{t, i}$ and a receiver $\mathrm{D}_{r, i}$ communicating in the mmWave channels. The baseband received signal by $\mathrm{D}_{r, i}$ can be written as follows:

where $\xi$ denotes the D2D distance parameter. Thus, the potential D2D receiver is randomly located around its transmitter according to a two-dimensional Gaussian distribution, resulting in (2) 30, 24]. The selection of the mode (D2D or cellular) for UE $i$ takes into account both $\delta_{i}$ and $L_{i}$. If UE $i$ is a potential DUE (i.e. $\delta_{i}=1$ ), it is considered as a DUE only if $L_{i} \leq \mu$, with $\mu$ corresponding to the D2D mode selection threshold (that is function of the wavelength, the attenuation of the signal and the sensitivity of the devices). Then, the potential D2D UEs in D2D mode form a $\mathrm{PPP} \Phi_{D}$ with intensity $\lambda_{D}=q \lambda \mathbb{P}(L<\mu)=q \lambda\left(1-e^{-\xi \pi \mu^{2}}\right)$.$$
\mathrm{D}_{r, i} \text { can be witten as follows: }
$$ 


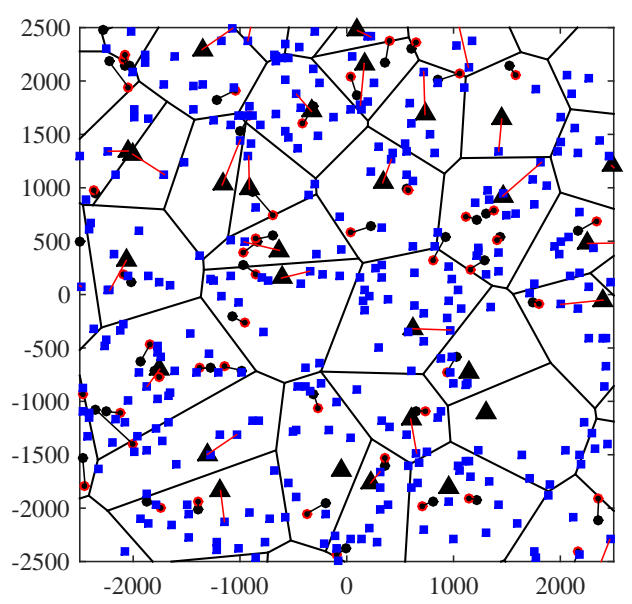

Figure 1: Network model taken into account in our work. Black triangles, blue squares, black dots and red dots denote BSs, CUEs, receiver DUEs and transmitter DUEs.

$$
\begin{aligned}
Y_{i}[n]= & \sqrt{P_{i} L_{i}^{-\alpha} g_{t, i} g_{r, i} h_{i}} S_{i}[n] \\
& +\sum_{X_{j} \in \Phi_{D} \backslash\left\{X_{i}\right\}} \sqrt{P_{j}\left\|X_{j}-X_{i}\right\|^{-\alpha} g_{t, j} g_{r, i} h_{i \leftarrow j}} S_{j}[n] \\
& +Z[n],
\end{aligned}
$$

where $P_{i}, L_{i}, g_{t, i}, g_{r, i}, h_{i}$ and $S_{i}$ denote the transmit power of $\mathrm{D}_{t, i}$, the distance between $\mathrm{D}_{t, i}$ and $\mathrm{D}_{r, i}$, the $i$-th transmitter device antenna gain, the $i$-th receiver device antenna gain, the channel fading and the unit-variance signal. Besides, $P_{j}, g_{t, j}, h_{i \leftarrow j}$ and $S_{j}$ denote the transmit power of the $j$-th transmitter, the $j$-th transmitter antenna gain, the channel fading of the link from the $j$-th transmitter to the $i$-th receiver and the unit-variance signal sent by the $j$-th transmitter.

In mmWave bands, it is generally assumed that small-scale fading is modeled by Nakagami-m fading [20, 31]. Nevertheless, recent works like [32, 33, show that treating the small-scale fading as Rayleigh gives relatively close results, and maintain key design insights. Then, in all this paper, we consider Rayleigh 
fading, i.e. $h_{i} \sim \operatorname{Exp}(1)$ and $h_{i \leftarrow j} \sim \operatorname{Exp}(1)$.

We also assume that fadings are independent over space. $\left\|X_{j}-X_{i}\right\|$ denotes the distance between the $j$-th transmitter and the $i$-th receiver. $Z[n]$ denotes the additive white Gaussian noise (AWGN).

In the case of Outband D2D communications, the interference for each receiver device come from the other D2D transmitters. We consider a single D2D link. As the $\operatorname{PPP} \Phi_{D}$ is stationary, we can assume that the receiver is located at the origin $o$ [24, 34] (the location of the receiver device is denoted $X_{o}=(0,0)$ ). Then, using Slivnyak's theorem, the total interference $I_{D}$ at a given D2D receiver is:

$$
I_{D}=\sum_{X_{j} \in \Phi_{D} \backslash\{o\}} P_{j} g_{t, j} g_{r, o} h_{j}\left\|X_{j}\right\|^{-\alpha} .
$$

The SINR of the typical D2D link can be written as

$$
\mathrm{SINR}_{\mathrm{D}}=\frac{P_{u, o}}{I_{D}+P_{N, o}}
$$

where $P_{u, o}=P_{o} g_{t, o} g_{r, o} L_{o}^{-\alpha} h_{o}$ and $P_{N, o}=N_{0} B_{w}$ (with $B_{w}=1 \mathrm{MHz}$ the signal bandwidth and $N_{0}=-174 \mathrm{dBm} / \mathrm{Hz}$ the noise power spectral density) denote the power of the typical link signal received by UE $o$ and the power of the noise at the $o$-th device, respectively.

\section{MM-Waves and Directional Antennas}

We assume that the Outband D2D communications use the mmWave spectrum, with the help of directional antennas. According to the results given in [35] and [1], we consider that the operating frequency is $f_{w}=28 \mathrm{GHz}$ (then the wavelength is $\lambda_{w}=10.7 \mathrm{~mm}$ ).

In the following of this paper, we propose to analyze the influence of directional antennas on the SINR and the data rates in Outband D2D communications. Directional antennas are divided in three types: patch antennas, horn antennas, and ULA-N (uniform linear array antennas with $N$ isotropic antennas). We introduce the gain functions $G_{p}$, with $p=0,1,2, N 2, N 5 . G_{p}$ denotes 
the ratio between the signal intensity in direction $\theta$, and the signal intensity with the same radiated power using an isotropic antenna $G_{0}(\theta)=1$ for $\theta \in[0,2 \pi]$. The significance of $p$ is denoted in the following sections.

Note that in all this paper, we propose to normalize the gain functions w.r.t. the maximum directivity (i.e. for $\theta=0$ degree).

\section{1. mmWave Directional Antennas}

First, we substitute the isotropic antenna model by two types of directional antennas with variable beam-width [21]: patch and horn antennas. The gain profiles for patch and horn antennas are theoretically defined as $G_{1}(\theta)=\frac{1+\cos \theta}{2}$ for $\theta \in[0,2 \pi]$ and $G_{2}(\theta)=\sin ^{2}\left(\theta-\frac{\pi}{2}\right)$ for $\theta \in\left[-\frac{\pi}{2}, \frac{\pi}{2}\right]$, respectively [36].

The gain profiles for $G_{1}$ and $G_{2}$ are depicted in Figure 2 (a) and (b), respectively.

\section{2. mmWave Antenna Arrays}

We also consider a ULA composed of $N$ isotropic antennas [37] at both the transmitter and the receiver (we call it ULA-N for simplification).

All the elementary isotropic antennas composing the array are separated by a distance $d$. The angle of departure of the mmWaves to the receiver is denoted as $\theta$. The array factor $A F(\theta, N, d)$ for an $N$-antenna array is defined by

$$
A F(\theta, N, d)=\sum_{n=1}^{N} a_{n} e^{j(n-1)(k d \cos \theta)},
$$

where $k=2 \pi / \lambda_{w}$ denotes the wave vector [36], and $a_{n}$ denotes the excitation of the $n$-th antenna element 38 . We propose to focus on ULA whose elements are mechanically aligned, i.e. beam-steering is not considered. Thus all the elements are identically excited: $\forall n \in[1, N], a_{n}=1$.

We consider that the reference point is the physical center of the ULA. Then, the radiation pattern $\zeta(\theta, N, d)$ of the array factor can be expressed as follows:

$$
\zeta(\theta, N, d)=\left|\frac{\sin (N k d \cos (\theta) / 2)}{\sin (k d \cos (\theta) / 2)}\right|
$$


In this work, $d=\lambda_{w} / 2=5.35 \mathrm{~mm}$. Note that we do not consider coupling effect for antenna arrays. Indeed, as shown by the simulation results depicted in [39, the coupling effect in ULA has a weak impact on the radiation pattern of such antennas.

In terms of power, $\zeta^{2}(\theta, N, d)$ represents the directivity of the array, which is due to the fact that elementary antennas are omni-directional Then, for $N=2$ and $N=5$, the normalized gain functions $G_{N 2}(\theta)=\frac{1}{2} \zeta^{2}\left(\theta, 2, \frac{\lambda_{w}}{2}\right)$ and $G_{N 5}(\theta)=\frac{1}{5} \zeta^{2}\left(\theta, 5, \frac{\lambda_{w}}{2}\right)$.

The gain profiles (in terms of power) are shown in Figure 2

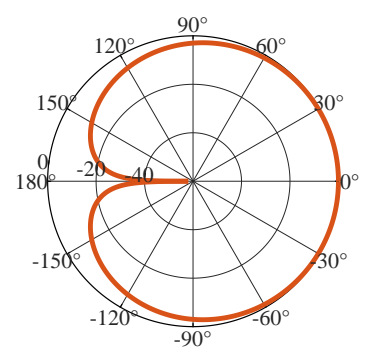

(a) $G_{1}$

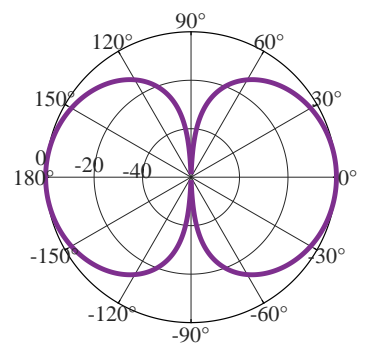

(c) $G_{N 2}$

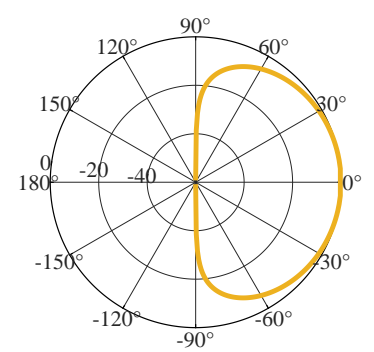

(b) $G_{2}$

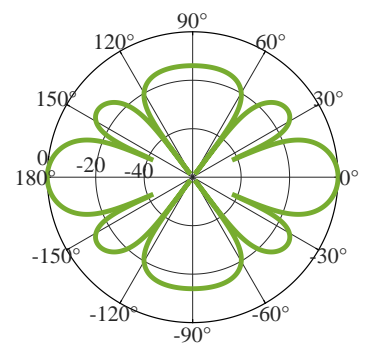

(d) $G_{N 5}$

Figure 2: Normalized radiation patterns of directional antennas (in dB).

\subsection{SINR with mmWaves}

215

\subsubsection{Interferences Characterization}

In this work, DUEs are communicating in the Outband, then the only interference felt by DUEs are those inherited from the other DUEs. 
We assume that for each D2D pair, the receiver's and the transmitter's antennas are perfectly aligned. This means that for each D2D pair, the power gain relative the antennas equals 1 .

Figure 3 shows graphically the interference in a two D2D pair network. In this simple configuration, $\mathrm{DUE}_{1}$ pair interferes with $\mathrm{DUE}_{2}$, and reciprocally. In the case of standard omni-directional antennas, the received interfering signal power only depends on the sending power and the distance between the interfering transmitter and the receiver (e.g. $\mathrm{DUE}_{t, 1}$ and $\mathrm{DUE}_{r, 2}$ ). However, as explained in [40], the received interfering signal power depends on

- the transmitting power (e.g. $\left.P_{1}\right)$

- the distance between the transmitter and the receiver (e.g. $L_{2,1}$ )

- the angle of departure (AOD) of the interfering signal (e.g. $\theta_{1,2}$ ) w.r.t. the $\mathrm{DUE}_{1}$ angle.

- the angle of arrival (AOA) of the interfering signal (e.g. $\theta_{2,1}$ ) w.r.t. the $\mathrm{DUE}_{2}$ angle.

- the radiation patterns of both the transmitter and the receiver (e.g. $G_{p}\left(\theta_{1,2}\right)$ and $\left.G_{p}\left(\theta_{2,1}\right)\right)$.

- the Rayleigh fading between the transmitter and the receiver (e.g. $h_{2 \leftarrow 1}$ ).

In particular, the power of the interference received by $\mathrm{DUE}_{r, 2}$ can be explained as follows:

$$
\begin{aligned}
P_{I_{2}} & =P_{r, 2 \leftarrow 1} \\
& =h_{2 \leftarrow 1} G_{p}\left(\theta_{1,2}\right) G_{p}\left(\theta_{2,1}\right) P_{1} L_{2,1}^{-\alpha}
\end{aligned}
$$

\section{Generalization}

We consider a vectorial basis with $\overrightarrow{0 x}$ and $\overrightarrow{0 y}$ axis. The oriented angle $\theta_{i}$ corresponds to the angle $\left(\overrightarrow{0 x}, \theta_{i}\right)$ (e.g. $\theta_{1}$ and $\theta_{2}$ in Figure 33. The oriented angle between the transmitted signal from $\mathrm{DUE}_{t, i}$ and the receiver of the $j$-th D2D pair, i.e. $\mathrm{DUE}_{r, j}$, is denoted as $\theta_{i, j}$. Similarly, $\theta_{j, i}$ corresponds to the angle 


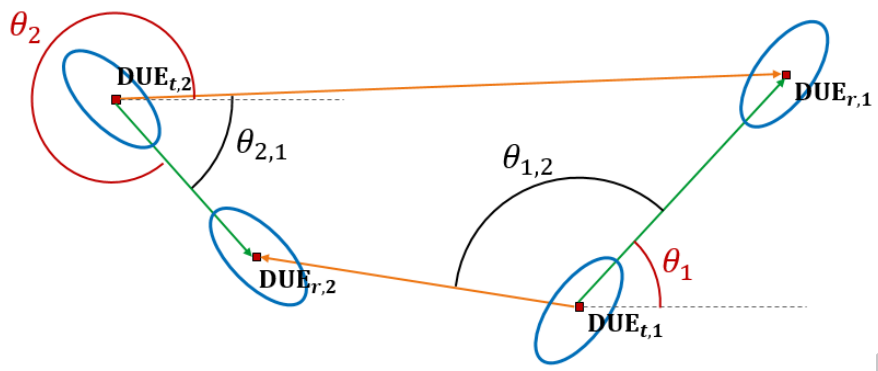

Figure 3: Interferences in a two-pair D2D network. Red squares, green lines, orange lines and blue lines represent DUEs, paired communications, interference and simplified radiation pattern.

between the transmitted signal from $\mathrm{DUE}_{t, j}$ and the receiver of the $i$-th $\mathrm{D} 2 \mathrm{D}$ pair, i.e. $\operatorname{DUE}_{r, i}$.

Thus, the total interference for the DUE $i$ is:

$$
I_{i}=\sum_{X_{j} \in \Phi_{D} \backslash\left\{X_{i}\right\}} h_{i \leftarrow j} G_{p}\left(\theta_{i, j}\right) G_{p}\left(\theta_{j, i}\right) P_{t, j} L_{i, j}^{-\alpha} .
$$

Using Slivnyak's theorem [34, the total interference for each DUE can be written as follows:

$$
I_{D, m m}=\sum_{X_{j} \in \Phi_{D} \backslash\{o\}} h_{o \leftarrow j} G_{p}\left(\theta_{o, j}\right) G_{p}\left(\theta_{j, o}\right) P_{t, j} L_{o, j}^{-\alpha} .
$$

\subsubsection{Coverage Probability}

With the help of (5), the SINR of a typical D2D link with directional mmWave antennas can be expressed as

$$
\operatorname{SINR}_{\mathrm{D}}=\frac{h_{o} G_{p}\left(\theta_{o, o}\right) G_{p}\left(\theta_{o, o}\right) P_{t, o} L_{o}^{-\alpha}}{\sum_{X_{j} \in \Phi_{D} \backslash\{o\}} h_{o \leftarrow j} G_{p}\left(\theta_{o, j}\right) G_{p}\left(\theta_{j, o}\right) P_{t, j} L_{o, j}^{-\alpha}+P_{N, o}} .
$$

As the receiver's and the transmitter's antennas of each D2D pair are aligned, 250 $G_{p}\left(\theta_{o, o}\right)=1$.

Moreover, we consider power channel inversion in this paper. This implies that the transmit power is calculated with respect to (w.r.t.) the distance 
between the transmitter and the receiver (nevertheless, it does not take into account the fading):

$$
G_{p}\left(\theta_{o, o}\right) G_{p}\left(\theta_{o, o}\right) P_{t, o} L_{o}^{-\alpha}=1 .
$$

$$
\begin{aligned}
\mathbb{P}\left(\operatorname{SINR}_{\mathrm{D}} \geq x\right) & =\mathbb{P}\left(h_{o} \geq x\left(\sum_{X_{j} \in \Phi_{D} \backslash\{o\}} h_{o \leftarrow j} G_{p}\left(\theta_{o, j}\right) G_{p}\left(\theta_{j, o}\right) P_{t, j} L_{o, j}^{-\alpha}+P_{N}\right)\right) \\
& \stackrel{(a)}{=} \mathcal{L}_{P_{N}}(x) \mathcal{L}_{I_{D, m m}}(x) \\
& \stackrel{(b)}{=} \exp \left(-P_{N} x-c_{m m} x^{\frac{2}{\alpha}}\right),
\end{aligned}
$$

Proposition 1. As explained before, we consider $G_{p}(\theta)$ the attenuation due to the directivity of the antennas. Then, the coverage probability for D2D links can be calculated as

$$
\mathrm{SNR}_{i}=\frac{P_{t, i} L_{i}^{-\alpha}}{N_{0} B_{w}}
$$

and thus $P_{N, i}=\frac{1}{\mathrm{SNR}_{i}}$. Note that the power of noise is assumed to be similar for each device (i.e. $\left.P_{N, i}=P_{N}, \forall i\right)$.

Subsequently, (11) can be written as

$$
\operatorname{SINR}_{\mathrm{D}}=\frac{h_{o}}{\sum_{X_{j} \in \Phi_{D} \backslash\{o\}} h_{o \leftarrow j} G_{p}\left(\theta_{o, j}\right) G_{p}\left(\theta_{j, o}\right) P_{t, j} L_{o, j}^{-\alpha}+P_{N}} .
$$

The Complementary Cumulative Distribution Function (CCDF) of the SINR (also known as Coverage Probability [41, 42]) representing the probability that the SINR is larger or equal to $x$ can be written [24]:

$$
\mathbb{P}\left(\operatorname{SINR}_{\mathrm{D}} \geq x\right)=\mathbb{P}\left(\frac{h_{o}}{I_{D, m m}+P_{N}} \geq x\right)
$$




Table 1: Numerical values for $\mathbb{E}\left[\left(G_{p}(\theta)\right)^{\frac{2}{\alpha}}\right]$
\begin{tabular}{|l|r|r|}
\hline $\boldsymbol{p}$ & for $\boldsymbol{\alpha}=\mathbf{2 . 5}$ & for $\boldsymbol{\alpha}=\mathbf{3 . 5}$ \\
\hline \hline 0 & 1 & 1 \\
\hline 1 & 0.548 & 0.610 \\
\hline 2 & 0.269 & 0.305 \\
\hline $\mathrm{N} 2$ & 0.391 & 0.446 \\
\hline $\mathrm{N} 5$ & 0.179 & 0.237 \\
\hline
\end{tabular}

where

$$
c_{m m} \stackrel{(c)}{=} \frac{\mathbb{E}^{2}\left[\left(G_{p}(\theta)\right)^{\frac{2}{\alpha}}\right] q\left(\frac{\lambda}{\xi}-\left(\frac{\lambda}{\xi}+\lambda \pi \mu^{2}\right) e^{-\xi \pi \mu^{2}}\right)}{\operatorname{sinc}\left(\frac{2}{\alpha}\right)} .
$$

In (16), equality (a) comes from the independence between the noise and all the received signals. Equality (b) comes from the fact that all the parameters are independently distributed. Equality (c) comes from the fact that the angles between each device are equiprobably distributed. Thus, the mean values for all the angles between devices are the same for all the DUEs, and thus $\mathbb{E}\left[G_{p}\left(\theta_{1}\right)\right] \mathbb{E}\left[G_{p}\left(\theta_{2}\right)\right]=\mathbb{E}^{2}\left[G_{p}(\theta)\right]$. The numerical values of $\mathbb{E}\left[\left(G_{p}(\theta)\right)^{\frac{2}{\alpha}}\right]$ for two path-loss exponents $(\alpha=2.5$ and $\alpha=3.5)$ are given in Table 1 .

See Appendix A for details.

\section{Results and Discussions}

The simulations have been made with Matlab software. All the D2D pairs have been distributed over a 12 macro-cell space according to a Poisson Point Process. The empirical values have been found after 20.000 iteration MonteCarlo simulation. Table 2 gives the parameters used for the simulations in this paper. We propose to analyze the CCDF of the SINR for $\mathrm{SNR}=10 \mathrm{~dB}$ with the various types of antennas described previously, in a sparse network first, then in a dense network. Figures 4 and 5 show that the analytical and simulation results are very close, which proves the accuracy of the theoretical analysis. 
Table 2: Simulation Parameters

Density of macro-cells $\lambda_{B}$ $\left(\pi 500^{2}\right)^{-1} \mathrm{~m}^{-2}$

Density of UEs $\lambda$ (sparse network) $2 \times\left(\pi 500^{2}\right)^{-1} \mathrm{~m}^{-2}$

Density of UEs $\lambda$ (dense network)

$100 \times\left(\pi 500^{2}\right)^{-1} \mathrm{~m}^{-2}$

D2D distance parameter $\xi$ $10 \times\left(\pi 500^{2}\right)^{-1} \mathrm{~m}^{-2}$

Potential D2D UEs $q$

Path-loss exponent $\alpha$

Mode selection threshold $\mu$

$150 \mathrm{~m}$

Band frequency

$28 \mathrm{GHz}$

\subsection{Coverage Probability in Sparse Network}

Figure 4 shows the coverage probability for D2D links for mmWaves in a network with a density of UEs $\lambda=2 \times\left(\pi 500^{2}\right)^{-1} \mathrm{~m}^{-2}$. It can be considered as a sparse network as $\lambda$ is close to $\lambda_{B}$. In Figure 4 (a), i.e. in a sparse network with a high path-loss exponent $(\alpha=3.5)$, we clearly see that the SINR for directional and omni-directional antenna are very close. This very thin difference is mainly due to the low density of UEs. The quantity of interference is quite small, as the potential interfering UEs are very limited. Moreover, the distance between UEs is theoretically high, as $\mathbb{E}\left[R^{*}\left(x, \Phi_{D}\right)\right]=\frac{1}{2 \sqrt{\lambda_{D}}}[43$, with $R^{*}\left(x, \Phi_{D}\right)=\min _{x_{i} \in \Phi_{D}}\left\|x_{i}-x\right\|$. Then, the impact of interfering signals is negligible, even if all the UEs use omni-directional antennas. It can also be proven thanks to 16 and 17 , where the variation of $\mathbb{E}^{2}\left[\left(G_{p}(\theta)\right)^{\frac{2}{\alpha}}\right]$ is negligible compared to the remaining term of $c_{m m}$. Thus, we can conclude that in such situation, the antenna does not need to be very directive: a patch antenna or a ULA-2 may be sufficient for a decent spectral efficiency.

However, in an almost free space condition $(\alpha=2.5)$, the advantage of directional antennas is more visible. Figure 4 (b) shows the CCDF of SINR for D2D links with $\alpha=2.5$. In this configuration, the difference in SINR between 


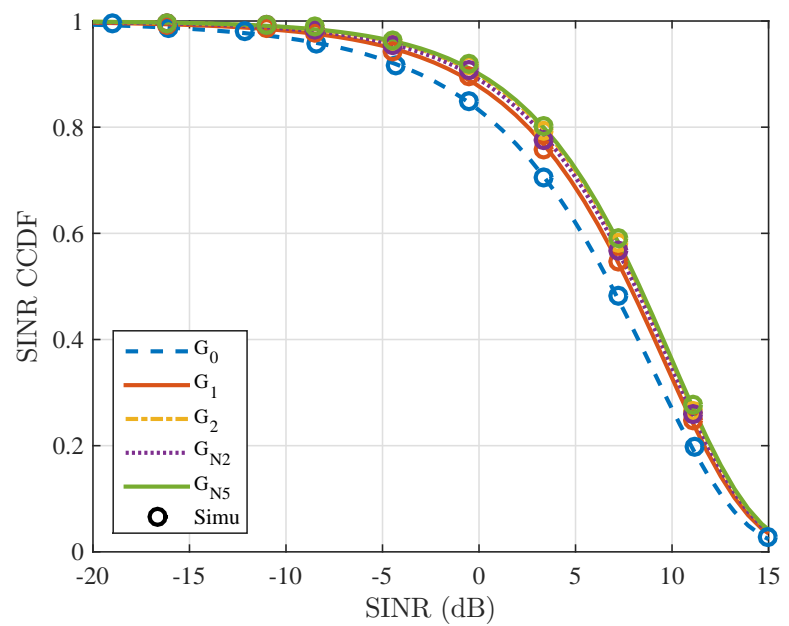

(a) $\alpha=3.5$

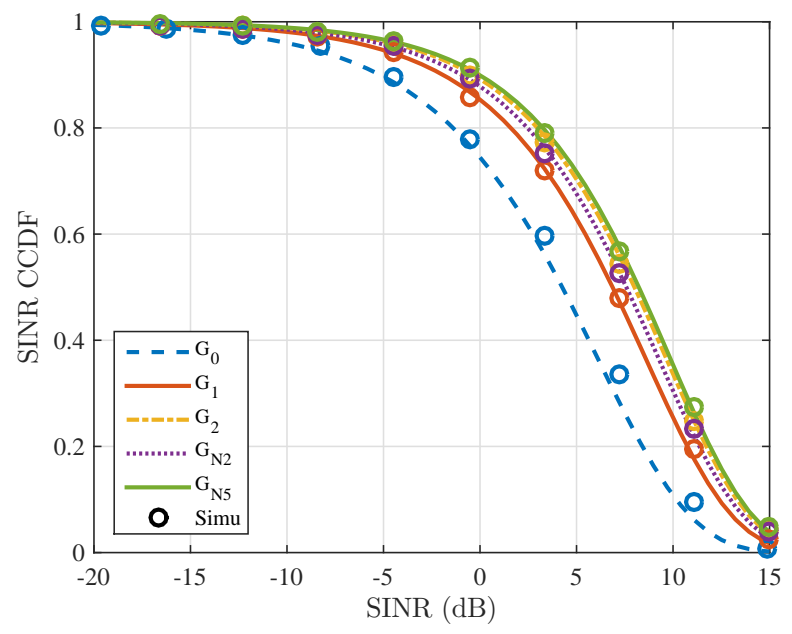

(b) $\alpha=2.5$

Figure 4: CCDF of SINR for D2D links with mmWaves in a sparse network, with $\lambda=$ $2 \times\left(\pi 500^{2}\right)^{-1} \mathrm{~m}^{-2}, \mathrm{SNR}=10 \mathrm{~dB}$ for all antenna types. 
omni-directional and directional antennas is clearer than with $\alpha=3.5$. This is mainly due to the low signal losses in the propagation channel. In fact, as the signals are being propagated further than in the previous case (for all antennas), the amount of interference is getting higher for poorly-directional network, with $\lambda=100 \times\left(\pi 500^{2}\right)^{-1} \mathrm{~m}^{-2}$, and $\alpha=3.5$.

In a dense network with $\alpha=3.5$, the SINR for directional antennas is far better than for omni-directional antenna. This difference is due to the high density of UEs in the network (and thus a very small distance between UEs), leading to a large amount of interference. Obviously, the SINR with omnidirectional antennas is way worse than in a sparse network (i.e. compared to the results in Figure 4). Indeed, for instance, $\mathbb{P}\left(\operatorname{SINR}_{\mathrm{D}} \geq 0 \mathrm{~dB}\right)=0.86$ in a sparse network and $\mathbb{P}\left(\mathrm{SINR}_{\mathrm{D}} \geq 0 \mathrm{~dB}\right)=0.28$ in a dense network with omnidirectional antennas. However, with directional antennas, the SINR is much 330

antennas, and each device has a worse impact on the others. Moreover, the amount of interference differs between all the directional antenna types. The difference is directly linked to the radiation patterns of the antennas shown in Figure 2 the more directional the antenna is, the fewer interference the network feels, and thus, the higher the SINR is. We clearly see that in terms of SINR, the worst antenna type is the omni-directional antenna $\left(G_{0}\right)$, that is radiating the same way all around itself (and thus leading to a high amount of interference). The patch antenna $\left(G_{1}\right)$ and the ULA-2 $\left(G_{N 2}\right)$ are more spectrally efficient than the omni-directional antenna, but do not lead to a better SINR than horn antennas $\left(G_{2}\right)$ and ULA-5 $\left(G_{N 5}\right)$, that are even more directional and lead to Nevertheless, the difference between all the directional antennas being quite thin, it is more reasonable to use the antenna with the most easy conception process, i.e. the patch antenna.

\subsection{Coverage Probability in Dense Network}

Figure 5 shows the CCDF of SINR for D2D links for mmWaves in a dense higher, with a maximum gain of $16 \mathrm{~dB}$ compared to omni-directional antennas. 


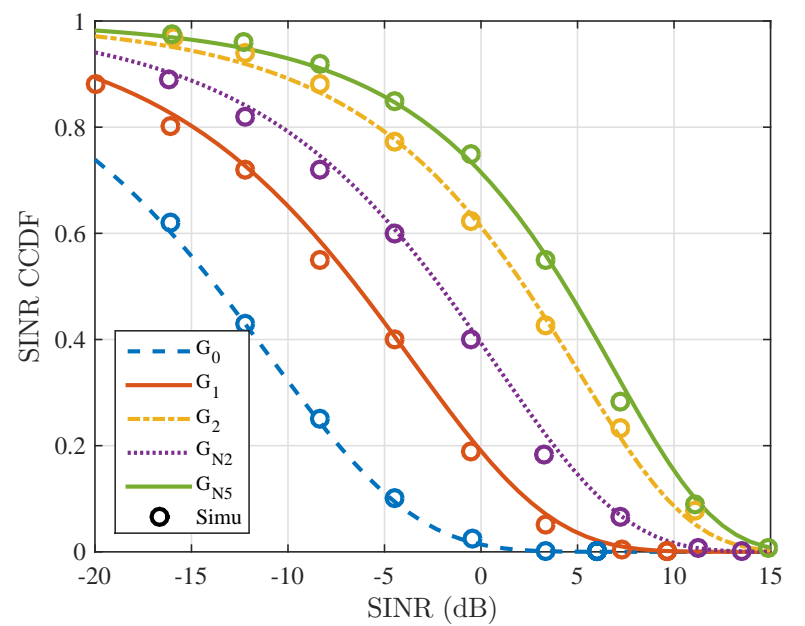

(a) $\alpha=3.5$

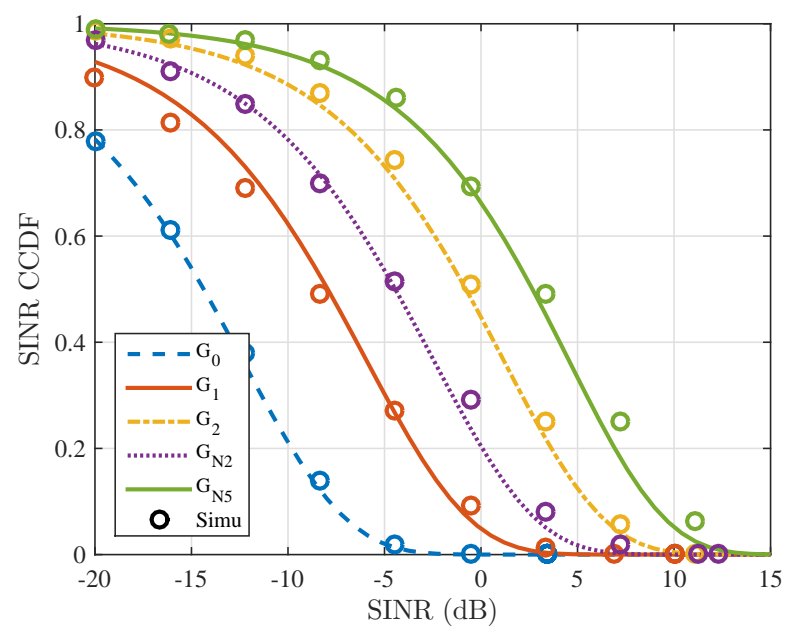

(b) $\alpha=2.5$

Figure 5: CCDF of SINR for D2D links with mmWaves in a dense network, with $\lambda=100 \times$ $\left(\pi 500^{2}\right)^{-1} \mathrm{~m}^{-2}, \mathrm{SNR}=10 \mathrm{~dB}$ for all antenna types. 
Nevertheless, we can see that the use of ULA-5 is not so efficient compared to horn antennas. The maximum difference between both types is $1 \mathrm{~dB}$ in such situation. Thus, we would prefer the horn antenna (that is easier to produce and integrate than the ULA-5).

In the case of an almost free-space environment (i.e. with $\alpha=2.5$ ), the SINR is obviously getting lower (which is due to a higher propagation of the signals compared to the previous case with $\alpha=3.5$ ), but the difference between each antenna is larger (like in the sparse network configuration with $\alpha=2.5$ ). However, the difference between the omni-directional and the most directional antennas is quite similar to the previous case. If we compare the ULA-5 and the horn antenna, we can see that the difference between both types is bigger than with $\alpha=3.5$, with a maximum of 3dB. In such situation, the use of ULA-5 could be of greater interest than other directional antenna types.

\subsection{Spectral Efficiency and Average Data Rate}

We define the ergodic link spectral efficiency $R$ as follows [42]:

$$
R=\mathbb{E}[\Delta \log (1+\mathrm{SINR})][\mathrm{bit} / \mathrm{s} / \mathrm{Hz}],
$$

where $\Delta$ denotes the frequency resources partition accessed by the typical link. $R$ combines modulation and coding schemes in the physical layer and multiple access protocols in the MAC layer. We introduce the spectral efficiency $R_{D}$ of D2D links as $R_{D}=\mathbb{E}\left[\Delta \log \left(1+\mathrm{SINR}_{\mathrm{D}}\right)\right]$ (in bit/s/Hz) [4]. As the resources accessed by the D2D links in Outband correspond to $100 \%$ of the total frequency and time resources, $R_{D}=\mathbb{E}\left[\log \left(1+\operatorname{SINR}_{\mathrm{D}}\right)\right]=\int_{0}^{\infty} \frac{e^{-P_{N} x}}{1+x} e^{-c_{m m} x^{\frac{2}{\alpha}}} \mathrm{d} x$.

The normalized average bit rate $T_{D}$ of D2D UEs is described in 24] as follows:

$$
\begin{aligned}
T_{D} & =\mathbb{P}(D<\mu) R_{D} \\
& =\left(1-e^{-\xi \pi \mu^{2}}\right) \int_{0}^{\infty} \frac{e^{-P_{N} x}}{1+x} e^{-c_{m m} x^{\frac{2}{\alpha}}} \mathrm{d} x .
\end{aligned}
$$




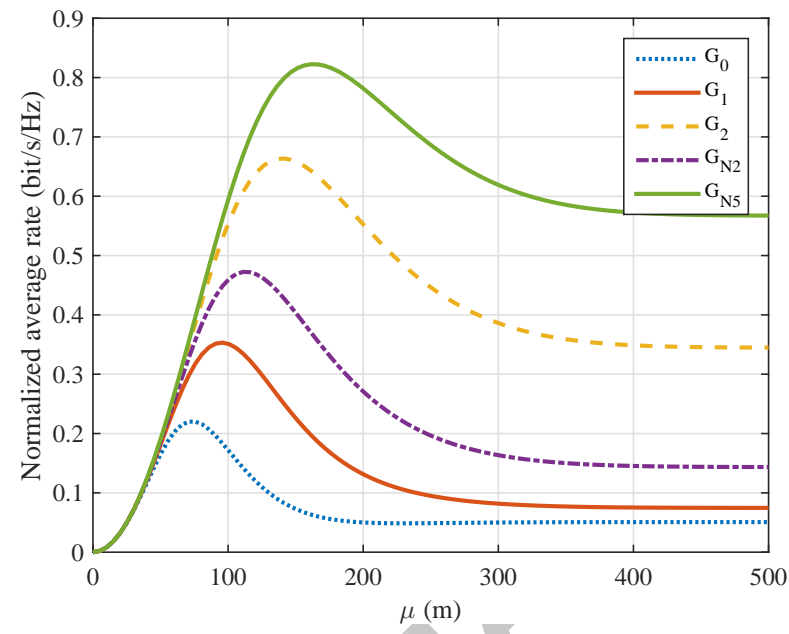

(a) $\alpha=3.5$

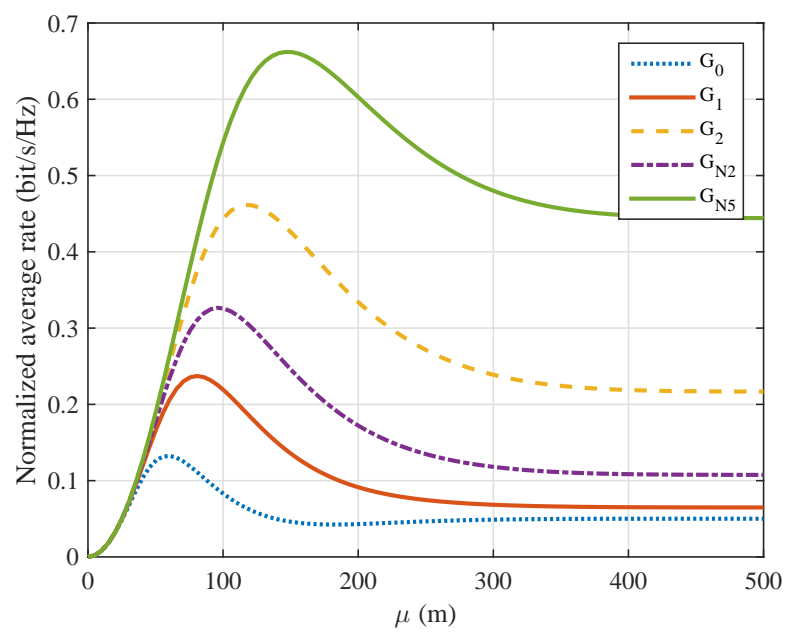

(b) $\alpha=2.5$

Figure 6: Normalized average ergodic data rate for $\alpha=3.5$ (a) and $\alpha=2.5$ (b), with $\lambda=100 \times\left(\pi 500^{2}\right)^{-1} \mathrm{~m}^{-2}$ for all antenna types. 
Figure 6 shows the average rates of potential D2D UEs as a function of D2D antennas such as horn antennas or ULA-5 improve considerably the SINR of the D2D links. Subsequently, the average data rate of D2D UEs is also improved with these types of antennas. Nevertheless, in sparse network, the use of very directional antennas is not really of great interest, as the density of UEs is small, and thus the SINR (and the data rates) does not highly differ from the case of omni-directional antennas.

This work can be further extended to an intelligent choice of antenna by the 
device itself, with regards to the environmental conditions. The choice implies a few parameters: the directivity of the antenna, the cost of the antenna, the targeted data rate (and thus the use of D2D). Of course, in terms of data rate, the more directional antennas are, the better the spectral efficiency is Nevertheless, with regards to the different use cases of D2D, it is not always mandatory to have very directional antennas such as ULA-5. Thus, a smart choice taking into account the aforementioned parameters would be of great interest. This choice could be made with optimization tools such as genetic algorithms, for instance.

\section{Acknowledgment}

This work was supported by RFS company through University of Nantes Foundation, Région Pays-de-la-Loire, Conseil Départemental de la Vendée and La Roche-sur-Yon Agglomération.

\section{References}

[1] M. Agiwal, A. Roy, N. Saxena, Next Generation 5G Wireless Networks: A Comprehensive Survey, IEEE Communications Surveys \& Tutorials 18 (3) (2016) 1617 - 1655. doi:10.1109/COMST.2016.2532458.

[2] A. Gupta, R. K. Jha, A Survey of 5G Network: Architecture and Emerging Technologies, IEEE Access 3 (2015) 1206-1232. doi:10.1109/ACCESS. 2015.2461602 .

[3] M. N. Tehrani, M. Uysal, H. Yanikomeroglu, Device-to-device communication in 5G cellular networks: challenges, solutions, and future directions, IEEE Communications Magazine 52 (5) (2014) 86-92. doi: 10.1109/MCOM.2014.6815897.

[4] R. Chevillon, G. Andrieux, J. F. Diouris, Energy Optimization of D2D Communications Using Relay Devices and Data Entropy, in: 2017 IEEE 
28th Annual International Symposium on Personal, Indoor, and Mobile

Radio Communications (PIMRC), Montréal, Canada, 2017, pp. 1-5. doi: 10.1109/PIMRC.2017.8292342.

[5] Y. Niu, C. Gao, Y. Li, L. Su, D. Jin, A. V. Vasilakos, Exploiting deviceto-device communications in joint scheduling of access and backhaul for mmwave small cells, IEEE Journal on Selected Areas in Communications 33 (10) (2015) 2052-2069. doi:10.1109/JSAC.2015.2435273

[6] Y. Niu, Y. Liu, Y. Li, X. Chen, Z. Zhong, Z. Han, Device-to-device communications enabled energy efficient multicast scheduling in mmwave small cells, IEEE Transactions on Communications 66 (3) (2018) 1093-1109. doi:10.1109/TCOMM.2017.2773529.

[7] A. Asadi, Q. Wang, V. Mancuso, A survey on device-to-device communication in cellular networks, IEEE Communications Surveys and Tutorials 16 (4) (2014) 1801-1819. doi:10.1109/COMST .2014.2319555.

[8] S. Xu, C. Xia, K. S. Kwak, Overlapping coalition formation games based interference coordination for D2D underlaying LTE-A networks, AEU International Journal of Electronics and Communications 70 (2) (2016) 204 - 209, doi:https://doi.org/10.1016/j.aeue.2015.10.007.

[9] A. Afzal, S. Ali, R. Zaidi, D. McLernon, M. Ghogho, On the Analysis of Device-to-Device Overlaid Cellular Networks in the Uplink under 3GPP Propagation Model, in: IEEE Wireless Communications and Networking Conference, 2016, pp. 1-6.

[10] J. Liu, N. Kato, J. Ma, N. Kadowaki, Device-to-Device Communication in LTE-Advanced Networks: A Survey, IEEE Communications Surveys and Tutorials 17 (4) (2015) 1923-1940. doi:10.1016/j.jnca.2016.06.004.

[11] A. Al-Hourani, S. Chandrasekharan, S. Kandeepan, Path loss study for 435 millimeter wave device-to-device communications in urban environment, 
2014 IEEE International Conference on Communications Workshops, ICC 2014 (2014) 102-107doi:10.1109/ICCW.2014.6881180.

[12] T. Wu, T. S. Rappaport, C. M. Collins, Safe for generations to come: Considerations of safety for millimeter waves in wireless communications,

IEEE Microwave Magazine 16 (2) (2015) 65-84. doi:10.1109/MMM.2014. 2377587 .

[13] W. Roh, J. Y. Seol, J. Park, B. Lee, J. Lee, Y. Kim, J. Cho, K. Cheun, F. Aryanfar, Millimeter-wave beamforming as an enabling technology for 5G cellular communications: theoretical feasibility and prototype results, IEEE Communications Magazine 52 (2) (2014) 106-113.

[14] M. Kyro, V. Kolmonen, P. Vainikainen, Experimental propagation channel characterization of mm-wave radio links in urban scenarios, IEEE Antennas Wireless Propagation Letters 11 (July) (2012) 865-868.

[15] M. Ji, G. Caire, A. Molisch, Wireless device-to-device caching networks: Basic principles and system performance, IEEE Journal on Selected Areas

口 in Communications 34 (1) (2013) 35. arXiv:1305.5216, doi:10.1109/ JSAC. 2015.2452672

[16] J. G. Andrews, S. Buzzi, W. Choi, S. V. Hanly, A. Lozano, A. C. K. Soong, J. C. Zhang, What Will 5G Be ?, IEEE Journal on Selected Areas in Communications 32 (6) (2014) 1065-1082.

[17] S. Niknam, A. A. Nasir, H. Mehrpouyan, B. Natarajan, A Multiband OFDMA Heterogeneous Network for Millimeter Wave 5G Wireless Applications, IEEE Access 4 (2016) 5640-5648. doi:10.1109/ACCESS.2016. 2604364

[18] T. Rappaport, R. Heath, R. Daniels, J. Murdock, Millimeter wave wireless communications, Prentice Hall, 2014. 
[19] J. Qiao, X. Shen, J. W. Mark, Q. Shen, Y. He, L. Lei, Enabling Device-toDevice Communications in Millimeter-Wave 5G Cellular Networks, IEEE Communications Magazine (January) (2015) 209-215.

[20] T. S. Rappaport, S. H. U. Sun, R. Mayzus, H. Zhao, Y. Azar, K. Wang, G. N. Wong, J. K. Schulz, M. Samimi, F. Gutierrez, Millimeter Wave Mobile Communications for 5G Cellular : It Will Work !, IEEE Access 1 (2013) 335-349.

[21] O. Georgiou, Simultaneous Wireless Information and Power Transfer in Cellular Networks with Directional Antennas, IEEE Communications Letters 21 (4) (2017) 885-888. doi:10.1109/LCOMM. 2016.2645562.

[22] M. Haenggi, J. G. Andrews, F. Baccelli, O. Dousse, M. Franceschetti, Stochastic Geometry and Random Graphs for the Analysis and Design of Wireless Networks, IEEE Journal on Selected Areas in Communications 27 (7) (2009) 1029-1046. doi:10.1109/JSAC.2009.090902.

[23] R. Chevillon, G. Andrieux, J.-F. Diouris, A comparison between Outband and Underlay Inband D2D communications, in: Sino-French workshop on information and communication technology (SFWICT), Qingdao, China, 2017.

[24] X. Lin, J. G. Andrews, A. Ghosh, Spectrum sharing for device-to-device communication in cellular networks, IEEE Transactions on Wireless Communications 13 (12) (2014) 6727-6740. arXiv:arXiv:1305.4219v4, doi: 10.1109/TWC.2014.2360202

[25] A. H. Sakr, E. Hossain, N. I. May, Cognitive and Energy Harvesting-Based D2D Communication in Cellular Networks : Stochastic Geometry Modeling and Analysis, IEEE Transactions on Communications 63 (5) (2015) 1-13. arXiv:arXiv:1405.2013v1, doi:10.1109/TCOMM.2015.2411266.

[26] M. Afshang, H. S. Dhillon, P. H. J. Chong, Modeling and Performance Analysis of Clustered Device-to-Device Networks, IEEE Transactions on 
Wireless Communications 15 (7) (2016) 4957-4972. doi:10.1109/TWC. 2016.2550024

[27] A. Al-Hourani, S. Kandeepan, A. Jamalipour, Stochastic geometry study on device-to-device communication as a disaster relief solution, IEEE Transactions on Vehicular Technology 65 (5) (2016) 3005-3017. doi: 10.1109/TVT.2015.2450223

[28] H. Elsawy, E. Hossain, On stochastic geometry modeling of cellular uplink transmission with truncated channel inversion power control, IEEE Transactions on Wireless Communications 13 (8) (2014) 4454-4469. arXiv: 1401.6145, doi:10.1109/TWC.2014.2316519.

[29] F. Baccelli, B. Blaszczyszyn, Stochastic Geometry and Wireless Networks, Vol. II, 2009. doi : oai:hal.archives-ouvertes.fr:inria-00403040_v4

[30] F. Baccelli, J. Li, T. Richardson, S. Subramanian, X. Wu, S. Shakkottai, On optimizing CSMA for wide area ad-hoc networks, 2011 International Symposium on Modeling and Optimization of Mobile, Ad Hoc, and Wireless Networks, WiOpt 2011 (2011) 354-359doi:10.1109/WIOPT. 2011.5930040

[31] K. Cho, J. Lee, C. G. Kang, Stochastic Geometry-based Coverage Analysis for Nakagami \& Log-normal Composite Fading Channel in Down-

口. link Cellular Networks, IEEE Communication Letters 7798 (99). doi: 10.1109/LCOMM. 2017.2669989.

[32] A. K. Gupta, J. G. Andrews, R. W. Heath, On the Feasibility of Sharing Spectrum Licenses in mmWave Cellular Systems, IEEE Transactions on Communications 64 (9) (2016) 3981-3995. arXiv:1512.01290, doi:10. 1109/TCOMM. 2016.2590467.

515 [33] Z. Jak, J. Ghosh, Network throughput and outage analysis in a poisson and matrn cluster based lte-advanced small cell networks, AEU - International 
Journal of Electronics and Communications 75 (2017) 46 - 52. doi:https: //doi.org/10.1016/j.aeue.2017.03.006

[34] D. Stoyan, W. S. Kendall, J. Mecke, Stochastic Geometry and its Applications, Wiley New York, 1995.

[35] G. R. Maccartney, J. Zhang, S. Nie, T. S. Rappaport, Path loss models for $5 \mathrm{G}$ millimeter wave propagation channels in urban microcells, GLOBECOM - IEEE Global Telecommunications Conference (2013) 39483953doi:10.1109/GLOCOM. 2013.6831690

[36] C. A. Balanis, Antenna Theory, Analysis and Design - Third Edition, Wiley New York, 2005.

[37] M. Soszka, S. Berger, A. Fehske, M. Simsek, B. Butkiewicz, G. Fettweis, Coverage and Capacity Optimization in Cellular Radio Networks with Advanced Antennas, in: WSA 2015; 19th International ITG Workshop on Smart Antennas, 2015, pp. 1-6.

[38] W. P. du Plessis, Efficient Computation of Array Factor and Sidelobe Level of Linear Arrays, IEEE Antennas \& Propagation Magazine 58 (6) (2016) $102-114$.

[39] D. Kelley, W. Stutzman, Array antenna pattern modeling methods that include mutual coupling effects, IEEE Transactions on Antennas and Propagation 41 (12) (1993) 1625-1632. doi:10.1109/8.273305.

[40] M. Soszka, S. Berger, M. Simsek, G. Fettweis, Energy Efficiency Optimization for 2D Antenna Arrays in Self-Organizing Wireless Networks, in: IEEE Wireless Communications and Networking Conference, 2016, pp. 1-7.

${ }_{540}$ [41] J. G. Andrews, T. Bai, M. Kulkarni, A. Alkhateeb, A. Gupta, R. W. Heath, Modeling and Analyzing Millimeter Wave Cellular Systems, IEEE Transactions on Communications 65 (1) (2017) 403-430. arXiv:1605.04283, doi:10.1109/TCOMM. 2016.2618794 
[42] H. ElSawy, A. Sultan-Salem, M.-S. Alouini, M. Z. Win, Modeling and Analysis of Cellular Networks Using Stochastic Geometry: A Tutorial, IEEE Communications Surveys \& Tutorials 19 (1) (2017) 167-203. arXiv: 1604.03689, doi:10.1109/COMST .2016.2624939.

[43] F. Baccelli, B. Blaszczyszyn, Stochastic Geometry and Wireless Networks, Vol. I, 2009. doi:10.1093/acprof : oso/9780199232574.003.0016

\section{Appendix A - Proof of Proposition 1}

Consider the conditional Laplace transform

$$
\begin{aligned}
\mathcal{L}_{I_{D, m m}}(x) & =\mathbb{E}\left[e^{-x I_{D, m m}}\right] \\
& \stackrel{(a)}{=} \mathbb{E}^{! o}\left[e^{-x \sum_{\mathbf{X}_{i} \in \Phi_{D}} P_{d, i} h_{i}\left\|X_{i}\right\|^{-\alpha} \varpi_{i} \varpi_{j}}\right] \\
& \stackrel{(b)}{=} \mathbb{E}\left[e^{-x \sum \sum_{X_{i} \in \Phi_{D}} P_{d, i} h_{i}\left\|X_{i}\right\|^{-\alpha} \varpi_{i} \varpi_{j}}\right]
\end{aligned}
$$

In equality (a), $\mathbb{E}^{! o}[\cdot]$ denotes the expectation with respect to the reduced Palm distribution, $\varpi_{i}=G_{p}\left(\angle \overrightarrow{D_{i j}}-\theta_{i}\right)$ and $\varpi_{j}=G_{p}\left(\angle \overrightarrow{D_{j i}}-\theta_{j}\right)$. Equality (b) comes from Slivnyak's theorem [34].

$$
\begin{aligned}
\mathcal{L}_{I_{D, m m}}(x) & \stackrel{(c)}{=} \exp \left(-\lambda_{D} \int_{0}^{2 \pi} \int_{0}^{\infty}\right. \\
& \left.\left(1-\mathbb{E}\left[\exp \left(-x P_{d, i} h_{i}\|r\|^{-\alpha} \varpi_{i} \varpi_{j}\right)\right]\right) r d r d \theta\right) \\
& =\exp \left(-\lambda_{D} \int_{0}^{2 \pi} x^{\frac{2}{\alpha}} \mathbb{E}\left[P_{d}^{\frac{2}{\alpha}}\right] \mathbb{E}\left[h^{\frac{2}{\alpha}}\right] \mathbb{E}\left[\left(\varpi_{i}\right)^{\frac{2}{\alpha}}\right]\right. \\
& \left.\mathbb{E}\left[\left(\varpi_{j}\right)^{\frac{2}{\alpha}}\right] \Gamma\left(1-\frac{2}{\alpha}\right) d \theta\right)
\end{aligned}
$$

555 Equality (c) comes from the probability generating functional of PPP [43]. 


$$
\begin{aligned}
\mathcal{L}_{I_{D, m m}}(x) \stackrel{(d)}{=} \exp \left(-\lambda_{D} \int_{0}^{2 \pi} x^{\frac{2}{\alpha}} \mathbb{E}\left[P_{d}^{\frac{2}{\alpha}}\right] \mathbb{E}^{2}\left[\left(\varpi_{i}\right)^{\frac{2}{\alpha}}\right]\right. \\
\left.\Gamma\left(1+\frac{2}{\alpha}\right) \Gamma\left(1-\frac{2}{\alpha}\right) d \theta\right) \\
=\exp \left(-\lambda_{D} \frac{x^{\frac{2}{\alpha}} \mathbb{E}\left[P_{d}^{\frac{2}{\alpha}}\right]}{\operatorname{sinc}\left(\frac{2}{\alpha}\right)} \int_{0}^{2 \pi} \mathbb{E}^{2}\left[\left(\varpi_{i}\right)^{\frac{2}{\alpha}}\right] d \theta\right)
\end{aligned}
$$

In equality (d), we have used $h \sim \operatorname{Exp}(1)$ and

$$
\begin{gathered}
\mathbb{E}\left[G_{p}\left(\angle \overrightarrow{D_{i j}}-\theta_{i}\right)\right]=\mathbb{E}\left[G_{p}\left(\angle \overrightarrow{D_{j i}}-\theta_{j}\right)\right] . \\
\mathcal{L}_{I_{D, m m}}(x) \stackrel{(e)}{=} \exp \left(-\lambda_{D} \frac{x^{\frac{2}{\alpha}}}{\operatorname{sinc}\left(\frac{2}{\alpha}\right)}\left(\frac{1}{\xi \pi}-\frac{\mu^{2} e^{-\xi \pi \mu^{2}}}{1-e^{-\xi \pi \mu^{2}}}\right)\right. \\
\left.\int_{0}^{2 \pi} \mathbb{E}^{2}\left[\left(\varpi_{i}\right)^{\frac{2}{\alpha}}\right] d \theta\right)
\end{gathered}
$$

In equality (e), we have used the value of $E\left[P_{d}^{\frac{2}{\alpha}}\right]$ demonstrated in [24].

$$
\begin{aligned}
\mathcal{L}_{I_{D, m m}}(x) & \stackrel{(f)}{=} \exp \left(-\lambda_{D} \frac{x^{\frac{2}{\alpha}}}{\operatorname{sinc}\left(\frac{2}{\alpha}\right)}\left(\frac{1}{\xi \pi}-\frac{\mu^{2} e^{-\xi \pi \mu^{2}}}{1-e^{-\xi \pi \mu^{2}}}\right)\right. \\
& \left.\mathbb{E}^{2}\left[\left(\varpi_{i}\right)^{\frac{2}{\alpha}}\right] \int_{0}^{2 \pi} d \theta\right) \\
& =\exp \left(-\lambda_{D} \frac{x^{\frac{2}{\alpha}}}{\operatorname{sinc}\left(\frac{2}{\alpha}\right)}\left(\frac{1}{\xi \pi}-\frac{\mu^{2} e^{-\xi \pi \mu^{2}}}{1-e^{-\xi \pi \mu^{2}}}\right)\right. \\
& \left.\frac{1}{2 \pi}\left(\int_{0}^{2 \pi}\left(G_{p}\left(\angle \overrightarrow{D_{i j}}-\theta_{i}\right)\right)^{\frac{2}{\alpha}} d \theta\right)^{2}\right)
\end{aligned}
$$

Finally, in equality (f), we have used the fact that $\mathbb{E}\left[\left(G_{p}\left(\theta_{1,2}\right)\right)^{\frac{2}{\alpha}}\right]$ does not depend on $\theta$. 